\title{
Survey for the Incidence of Tobacco streak virus (TSV) in Field and Horticultural Crops
}

\author{
M. Sunil Kumar ${ }^{1 *}$, R. Sarada Jayalakshmi Devi ${ }^{2}$, M. Krishna $\operatorname{Reddy}^{3}$, K. Vemana ${ }^{4}$, \\ T. Murali Krishna ${ }^{5}$ and L. Prasanthi ${ }^{6}$
}

${ }^{1}$ Department of Plant Pathology, S.V. Agricultural College, Tirupathi, 517 502, India

${ }^{2}$ University Librarian, Acharya N.G. Ranga Agricultural University, Guntur, 522 209, India

${ }^{3}$ Division of Plant Pathology, ICAR-IIIHR, Bangalore, 560089, India

${ }^{4}$ Department of Plant Pathology, Agricultural Research Station, Kadiri, India

${ }^{5}$ Department of Entomology, ${ }^{6}$ Department of genetics \& Plant Breeding, IFT, RARS,

Tirupathi, 517 502, India

*Corresponding author

\section{A B S T R A C T}

\begin{tabular}{|l|}
\hline K e y w o r d s \\
$\begin{array}{l}\text { Ilarvirus, TSV, Okra, } \\
\text { Gherkin, Cucumber, } \\
\text { Pumpkin }\end{array}$ \\
\hline Article Info \\
\hline $\begin{array}{l}\text { Accepted: } \\
\text { 26 September } 2018 \\
\text { Available Online: } \\
10 \text { October } 2018\end{array}$ \\
\hline
\end{tabular}

\section{Introduction}

TSV is the type member of Ilarvirus genus of the Bromoviridae family. In recent years, Ilarviruses (TSV) had received national and international attention because they cause major crop losses. TSV had caused severe epidemics in India on several important crops such as groundnut, sunflower, okra, cotton, cucurbits and gherkins. However, in recent years, vegetable crop cultivation (okra,

\begin{abstract}
Survey conducted for disease incidence of Tobacco streak virus (TSV) during Kharif 2014-15 and 2015-16 in Andhra Pradesh and Karnataka revealed disease incidence of 9-28 per cent in groundnut, 6-18 per cent in sunflower and 5-22 per cent in cucumber. In Andhra Pradesh during Kharif 2014-15 and 2015-16, the incidence of TSV in groundnut ranged from 9.8 (Bathalapalli) to 28.2 (Obuldevarayacheruvu) per cent and 9.2 (Kothacheruvu) to 23.2 (Gorantla) per cent respectively; similarly on sunflower the incidence ranged from 6.4 (Aluru) to 18.7 (Bethamcherla) per cent and 5.2 (Adoni) to 14.2 (Bethamcherla) per cent respectively. In Karnataka the incidence of TSV during Kharif 2014-15 and 2015-16 ranged from 10.9 (Herapanahalli) to 22.8 (Tumkur) per cent and 5.2 (Pavagada) to 14.2 (Tumkur) per cent respectively. The collected samples were tested by Enzyme Linked Immunosorbent Assay (ELISA) using TSV specific antisera and through electron microscopy and positive samples were maintained on cowpea for further studies.
\end{abstract}

gherkin, cucumber, pumpkin) has been seriously hampered due to TSV infection. Due to wide range of host plants, transmission through pollen and seed, this virus was placed among the most economically important virus. TSV infection at seedling stage results in premature death of the plant, Infection during mid - stage of the plant growth may result in necrosis of the leaves and severe reduction in yield. Infection at late stage of the plant growth results in mild chlorotic symptoms 
with little effect on plant growth and yield. In several weed hosts, such as parthenium, TSV causes asymptomatic infection. Keeping in view the economic importance of virus, a survey was conducted in Andhra Pradesh and Karnataka to assess the disease incidence of TSV in groundnut, sunflower and cucumber.

\section{Materials and Methods}

\section{Field surveys}

Roving survey was conducted to note the incidence of Tobacco Streak Virus (TSV) in different crop plants viz., groundnut, sunflower and Cucumber from different locations of Andhra Pradesh and Karnataka. The Suspected TSV infected samples exhibiting characteristic symptoms of severe necrosis of leaf lamina, petioles and other plant parts were collected and identified by Enzyme Linked Immunosorbent Assay (ELISA) using TSV specific antisera or through electron microscopy by leaf dip method. The TSV identified samples either stored in $-80^{\circ} \mathrm{C}$ for further virus characterization or mechanically inoculated to test hosts for culture maintenance in an insect proof glass house.

\section{Electron microscopy}

The suspected plant sample was ground in Sodium phosphate buffer pH 7.5 at 1:5 (w/v) proportion filtered through double layered cheese cloth and observed under transmission electron microscope for determining the shape and size of the virus particles causing mosaic symptoms on diseased sample. To examine virus particles in suspected leaf, a drop of filtered leaf preparation was placed on the carbon-coated grids and allowed to settle for 2-3 minutes. The excess of sample was removed by using blotting paper. A small droplet of dye (uranyl acetate or phosphotungstic acid) was placed on it and allowed to stay for 2-3 minutes. The excess stain was drained by touching the blotting paper strip to the edge of the grid. The grids were dried for 15-30 $\mathrm{min}$ in dessicator and examined under JOEL $100 \mathrm{~S}$ transmission electron microscope at various magnifications. The photographs of the virus particles were taken.

Actual size of a Particle in $\mathrm{nm}=$ $\underline{\text { Size of the particle in electron micrograph }(\mathrm{mm})}$

$$
\text { Magnific ation }
$$

\section{Bioassay}

Suspected TSV infected samples from groundnut, sunflower and cucumber were collected from different locations of Andhra Pradesh and Karnataka, were used for sap inoculation on cowpea (Vigna unguiculata cv. C-152), a diagnostic host for TSV. Test plants were raised in insect proof glasshouse conditions. Five to eight seeds were planted in each plastic pot (4" diameter) and cotyledonary stage with uniform growth were selected for inoculation. Infected as well as healthy tissues from field samples macerated separately using sterilized chilled pestle and motor in $0.1 \mathrm{M}$ phosphate buffer $(\mathrm{pH} 7.2,1: 1$ $\mathrm{w} / \mathrm{v})$ containing $0.1 \%$ mercaptoethanol as extraction buffer (EB) (Appendix I). The sap was kept on ice till the inoculation was completed. The test plant seedlings were dusted with celite (diatomaceous earth) or carborandum powder, which served as an abrasive. The inoculum was applied directly on to the upper surface of the leaves. After inoculations, the seedlings were sprayed with distilled water and kept in the insect-proof glass house. Inoculations were preferably carried out in the evening. The inoculated seedlings were observed for symptoms development. Local lesions developed 4-5 days post-inoculation were used for further inoculation by taking single lesion and subsequently maintained pure virus cultures of 
groundnut, sunflower and cucumber TSV isolates on cowpea.

\section{Results and Discussion}

Intensive roving survey was conducted during Kharif 2014-15 and 2015-16 to record the disease incidence of TSV in groundnut, sunflower and cucumber at different places in Andhra Pradesh and Karnataka. The incidence of TSV in Andhra Pradesh ranged from 9-28 per cent in groundnut and 6-18 per cent in sunflower samples. The incidence of TSV in cucumber ranged from 5-22 percent in Tumkur and Davanagere districts of Karnataka. In Andhra Pradesh during Kharif 2014-15 the incidence of TSV in groundnut was highest in Obuldevaraya cheruvu (28.2 per cent) and lowest in Bathalapalli (9.8 per cent). Similarly Gorantla recorded highest disease incidence of 23.2 per cent and Kothacheruvu recorded lowest disease incidence of 9.2 per cent during Kharif 201516 (Table 1 and Fig. 1).

In sunflower, Bethamcherla recorded highest disease incidence of 18.7 and 14.2 per cent during Kharif 2014-15 and 2015-16 respectively and lowest disease incidence was recorded in Aluru (6.4 per cent) and Adoni (5.2 per cent) during Kharif 2014-15 and 2015-16 respectively (Table 2 and Fig. 2). In Karnataka, cucumber growing areas of Tumkur district, Sira recorded highest disease incidence of 22.8 (Kharif, 2014-15) and 14.2 (Kharif, 2015-16) per cent, while Herapanahalli (10.9 per cent) and Pavagada (5.2 per cent) recorded lowest disease incidence during Kharif 2014-15 and 2015-16 respectively (Table 3 and Fig. 3).

TSV incidence was first observed on groundnut during the year 2000 (Reddy et al., 2002) in Anantapur district of Andhra Pradesh. Since then its incidence on groundnut crop has been recorded regularly (16.5 per cent-2004; 5-80 per cent-2006 and 2-30 per cent-2007) in Andhra Pradesh (Anonymous 2004b, 2006 and 2007). TSV incidence has also been reported on groundnut in Raichur district of Karnataka (Prasada Rao et al., 2003). Sunflower Necrosis Disease (SND) caused by Tobacco streak virus was reported as an epidemic consecutively for the three years (1997-99), with the incidence ranging from 10 to 80 percent and causing yield losses up to 90 per cent in most of the sunflower growing regions of Southern India (DOR Annual Report, 2001). Halakeri (1999) recorded incidence of sunflower necrosis disease ranged from 3 to 70 per cent around Dharwad, Gadag, Bagalkot, Haveri and Bijapur districts. Five to thirty per cent necrosis disease was recorded at Sira, Chitradurga, Bellary, Bijapur and Gulbarga districts, 50 to 70 per cent around Nagalapur and Lingsur of Raichur district (Anonymous, 2002). Krishna Reddy et al., (2003) reported severe outbreak of Tobacco streak virus (TSV) in cucumber and gherkin causing yield losses of 31 to 75 per cent in Bangalore, Bellary, Davanagiree, and Tumkur districts of Karnataka state during 2000-2002.

\section{Identification of TSV isolates}

Suspected Tobacco streak virus (TSV) infected groundnut samples showing necrotic symptoms on leaves, top bud and stem collected from farmer's fields of different mandals in Anantapur district during Kharif, 2014-15 and 2015-16 were tested by bio assay and ELISA for the TSV identification. Out of 44 samples collected, 30 samples reacted positively with polyclonal antiserum to TSV by DAC-ELISA. The absorbance values $\left(\mathrm{A}_{405}\right.$ $\mathrm{nm}$ ) ranged from 0.19-3.16 (Table 1). TSV suspected symptomatic groundnut samples were sap transmitted to the cowpea seedlings (Vigna unguiculata cv. 132) and presence of TSV was confirmed by DAC-ELISA ( $\mathrm{A}_{405}$ nm: 2.45-3.12) (Table 4). 
Table.1 Per cent disease incidence of Tobacco streak virus (TSV) in groundnut samples and absorbance values (A $\left.\mathrm{A}_{405} \mathrm{~nm}\right)$ of samples collected from different mandals of Andhra Pradesh

\begin{tabular}{|c|c|c|c|c|c|c|c|}
\hline \multirow[t]{2}{*}{ District } & \multirow[t]{2}{*}{ Place (Mandal) } & \multicolumn{2}{|c|}{ Per cent disease incidence } & \multirow{2}{*}{$\begin{array}{l}\text { Sample } \\
\text { code }\end{array}$} & \multirow{2}{*}{$\begin{array}{c}\left(\mathrm{A}_{405}\right. \\
\mathrm{nm})\end{array}$} & \multirow{2}{*}{$\begin{array}{c}\text { Range of } \\
\text { absorbance } \\
\text { values }\left(\mathbf{A}_{405} \mathbf{n m}\right)\end{array}$} & \multirow{2}{*}{$\begin{array}{l}\text { Range of } \\
\text { disease } \\
\text { incidence } \\
(\%)\end{array}$} \\
\hline & & Kharif2014 & Kharif 2015 & & & & \\
\hline \multirow{20}{*}{$\begin{array}{l}\text { Ananthapur } \\
\text { (Andhra } \\
\text { Pradesh) }\end{array}$} & \multirow[t]{4}{*}{ Nallamada } & \multirow[t]{4}{*}{14.2} & \multirow[t]{4}{*}{22.2} & GNAP1 & 2.44 & \multirow[t]{20}{*}{$0.19-3.16$} & $9-28$ \\
\hline & & & & GNAP2 & 2.89 & & \\
\hline & & & & GNAP3 & 2.42 & & \\
\hline & & & & GNAP4 & 1.22 & & \\
\hline & \multirow[t]{4}{*}{ N.P. Kunta } & \multirow[t]{4}{*}{21.1} & \multirow[t]{4}{*}{19.8} & GNAP5 & 3.02 & & \\
\hline & & & & GNAP6 & 1.32 & & \\
\hline & & & & GNAP7 & 3.16 & & \\
\hline & & & & GNAP8 & 0.42 & & \\
\hline & \multirow{4}{*}{$\begin{array}{l}\text { Obuldevaraya } \\
\text { cheruvu }\end{array}$} & \multirow[t]{4}{*}{28.2} & \multirow[t]{4}{*}{11.2} & GNAP9 & 1.76 & & \\
\hline & & & & GNAP10 & 0.39 & & \\
\hline & & & & GNAP11 & 3.02 & & \\
\hline & & & & GNAP12 & 0.22 & & \\
\hline & \multirow[t]{4}{*}{ Kothacheruvu } & \multirow[t]{4}{*}{19.2} & \multirow[t]{4}{*}{9.2} & GNAP13 & 1.43 & & \\
\hline & & & & GNAP14 & 2.92 & & \\
\hline & & & & GNAP15 & 0.62 & & \\
\hline & & & & GNAP16 & 3.00 & & \\
\hline & \multirow[t]{4}{*}{ Rapthadu } & \multirow[t]{4}{*}{10.2} & \multirow[t]{4}{*}{16.6} & GNAP17 & 1.64 & & \\
\hline & & & & GNAP18 & 0.82 & & \\
\hline & & & & GNAP19 & 0.19 & & \\
\hline & & & & GNAP20 & 0.23 & & \\
\hline
\end{tabular}


Int.J.Curr.Microbiol.App.Sci (2018) 7(10): 3657-3669

\begin{tabular}{|c|c|c|c|c|c|c|c|}
\hline \multirow[t]{2}{*}{ District } & \multirow[t]{2}{*}{ Place (Mandal) } & \multicolumn{2}{|c|}{ Per cent disease incidence } & \multirow{2}{*}{$\begin{array}{l}\text { Sample } \\
\text { code }\end{array}$} & \multirow{2}{*}{$\begin{array}{l}\left(\mathrm{A}_{405}\right. \\
\mathrm{nm})\end{array}$} & \multirow{2}{*}{$\begin{array}{c}\text { Range of } \\
\text { absorbance } \\
\text { values (A } \mathbf{A}_{405} \\
\text { nm) }\end{array}$} & \multirow{2}{*}{$\begin{array}{c}\text { Range of } \\
\text { disease } \\
\text { incidence (\%) }\end{array}$} \\
\hline & & Kharif 2014 & Kharif 2015 & & & & \\
\hline \multirow{24}{*}{$\begin{array}{l}\text { Ananthapur } \\
\text { (Andhra } \\
\text { Pradesh) }\end{array}$} & \multirow[t]{4}{*}{ Kalluru } & \multirow[t]{4}{*}{14.6} & \multirow[t]{4}{*}{14.2} & GNAP21 & 0.71 & \multirow[t]{24}{*}{$0.19-3.16$} & \multirow[t]{24}{*}{$9-28$} \\
\hline & & & & GNAP22 & 2.65 & & \\
\hline & & & & GNAP23 & 1.12 & & \\
\hline & & & & GNAP24 & 2.72 & & \\
\hline & \multirow[t]{4}{*}{ Penukonda } & \multirow[t]{4}{*}{22.4} & \multirow[t]{4}{*}{19.8} & GNAP25 & 0.55 & & \\
\hline & & & & GNAP26 & 2.82 & & \\
\hline & & & & GNAP27 & 1.84 & & \\
\hline & & & & GNAP28 & 2.96 & & \\
\hline & \multirow[t]{4}{*}{ Gorantla } & \multirow[t]{4}{*}{25.2} & \multirow[t]{4}{*}{23.2} & GNAP29 & 1.24 & & \\
\hline & & & & GNAP30 & 2.62 & & \\
\hline & & & & GNAP31 & 1.92 & & \\
\hline & & & & GNAP32 & 2.02 & & \\
\hline & \multirow[t]{4}{*}{ Dharmavaram } & \multirow[t]{4}{*}{11.2} & \multirow[t]{4}{*}{10.2} & GNAP33 & 2.22 & & \\
\hline & & & & GNAP34 & 1.54 & & \\
\hline & & & & GNAP35 & 1.72 & & \\
\hline & & & & GNAP36 & 2.14 & & \\
\hline & \multirow[t]{4}{*}{ Bathalapalli } & \multirow[t]{4}{*}{9.8} & \multirow[t]{4}{*}{9.4} & GNAP37 & 0.56 & & \\
\hline & & & & GNAP38 & 1.92 & & \\
\hline & & & & GNAP39 & 0.62 & & \\
\hline & & & & GNAP40 & 0.45 & & \\
\hline & \multirow[t]{4}{*}{ Kothacheruvu } & \multirow[t]{4}{*}{13.2} & \multirow[t]{4}{*}{15.2} & GNAP41 & 1.67 & & \\
\hline & & & & GNAP42 & 2.01 & & \\
\hline & & & & GNAP43 & 0.22 & & \\
\hline & & & & GNAP44 & 0.12 & & \\
\hline
\end{tabular}


Table.2 Per cent disease incidence of Tobacco streak virus (TSV) in sunflower samples and absorbance values (A405 $\mathrm{nm}$ ) of samples collected from different mandals of Andhra Pradesh

\begin{tabular}{|c|c|c|c|c|c|c|c|}
\hline \multirow[t]{2}{*}{ District } & \multirow[t]{2}{*}{ Place (Mandal) } & \multicolumn{2}{|c|}{$\begin{array}{l}\text { Per cent disease } \\
\text { incidence }\end{array}$} & \multirow[t]{2}{*}{$\begin{array}{l}\text { Sample } \\
\text { code }\end{array}$} & \multirow[t]{2}{*}{$\left(\mathbf{A}_{405} \mathrm{~nm}\right)$} & \multirow{2}{*}{$\begin{array}{c}\text { Range of } \\
\text { Absorbance } \\
\text { values }\left(\mathbf{A}_{405} \mathbf{n m}\right)\end{array}$} & \multirow{2}{*}{$\begin{array}{c}\text { Range of } \\
\text { disease } \\
\text { incidence }(\%)\end{array}$} \\
\hline & & Kharif2014 & Kharif 2015 & & & & \\
\hline \multirow{20}{*}{$\begin{array}{l}\text { Kurnool } \\
\text { (Andhra } \\
\text { Pradesh) }\end{array}$} & \multirow[t]{4}{*}{ Bethamcherla } & \multirow[t]{4}{*}{18.7} & \multirow[t]{4}{*}{14.2} & SFAP1 & 2.62 & \multirow[t]{20}{*}{$0.11-2.76$} & \multirow[t]{20}{*}{$6-18$} \\
\hline & & & & SFAP2 & 1.28 & & \\
\hline & & & & SFAP3 & 0.52 & & \\
\hline & & & & SFAP4 & 1.43 & & \\
\hline & \multirow[t]{4}{*}{ Atmakur } & \multirow[t]{4}{*}{11.2} & \multirow[t]{4}{*}{10.2} & SFAP5 & 0.86 & & \\
\hline & & & & SFAP6 & 1.91 & & \\
\hline & & & & SFAP7 & 0.37 & & \\
\hline & & & & SFAP8 & 2.44 & & \\
\hline & \multirow[t]{4}{*}{ Aluru } & \multirow[t]{4}{*}{6.4} & \multirow[t]{4}{*}{7.4} & SFAP9 & 0.28 & & \\
\hline & & & & SFAP10 & 1.82 & & \\
\hline & & & & SFAP11 & 2.10 & & \\
\hline & & & & SFAP12 & 0.67 & & \\
\hline & \multirow[t]{4}{*}{ Nandikotkur } & \multirow[t]{4}{*}{9.2} & \multirow[t]{4}{*}{8.9} & SFAP13 & 1.55 & & \\
\hline & & & & SFAP14 & 0.32 & & \\
\hline & & & & SFAP15 & 1.62 & & \\
\hline & & & & SFAP16 & 0.28 & & \\
\hline & \multirow[t]{4}{*}{ Aspari } & \multirow[t]{4}{*}{17.2} & \multirow[t]{4}{*}{9.2} & SFAP17 & 2.76 & & \\
\hline & & & & SFAP18 & 1.37 & & \\
\hline & & & & SFAP19 & 1.42 & & \\
\hline & & & & SFAP20 & 2.44 & & \\
\hline
\end{tabular}


Int.J.Curr.Microbiol.App.Sci (2018) 7(10): 3657-3669

Contd...

\begin{tabular}{|c|c|c|c|c|c|c|c|}
\hline \multirow[t]{2}{*}{ District } & \multirow[t]{2}{*}{ Place (Mandal) } & \multicolumn{2}{|c|}{$\begin{array}{l}\text { Per cent disease } \\
\text { incidence }\end{array}$} & \multirow[t]{2}{*}{$\begin{array}{l}\text { Sample } \\
\text { code }\end{array}$} & \multirow[t]{2}{*}{$\left(\mathbf{A}_{405} \mathrm{~nm}\right)$} & \multirow{2}{*}{$\begin{array}{c}\text { Range of } \\
\text { Absorbance } \\
\text { values }\left(\mathbf{A}_{405}\right. \\
\mathbf{n m})\end{array}$} & \multirow{2}{*}{$\begin{array}{c}\text { Range of } \\
\text { disease } \\
\text { incidence } \\
(\%)\end{array}$} \\
\hline & & $\begin{array}{c}\text { Kharif } \\
2014\end{array}$ & Kharif 2015 & & & & \\
\hline \multirow{20}{*}{$\begin{array}{l}\text { Kurnool } \\
\text { (Andhra } \\
\text { Pradesh) }\end{array}$} & \multirow[t]{4}{*}{ Paparli } & \multirow[t]{4}{*}{12.9} & \multirow[t]{4}{*}{11.6} & SFAP21 & 1.28 & \multirow[t]{20}{*}{$0.11-2.76$} & \multirow[t]{20}{*}{$6-18$} \\
\hline & & & & SFAP22 & 2.62 & & \\
\hline & & & & SFAP23 & 1.62 & & \\
\hline & & & & SFAP24 & 2.46 & & \\
\hline & \multirow[t]{4}{*}{ Veldthurthi } & \multirow[t]{4}{*}{4.2} & \multirow[t]{4}{*}{13.2} & SFAP25 & 1.42 & & \\
\hline & & & & SFAP26 & 2.29 & & \\
\hline & & & & SFAP27 & 1.32 & & \\
\hline & & & & SFAP28 & 1.92 & & \\
\hline & \multirow[t]{4}{*}{ Adoni } & \multirow[t]{4}{*}{6.7} & \multirow[t]{4}{*}{5.2} & SFAP29 & 0.42 & & \\
\hline & & & & SFAP30 & 2.42 & & \\
\hline & & & & SFAP31 & 0.91 & & \\
\hline & & & & SFAP32 & 2.68 & & \\
\hline & \multirow[t]{4}{*}{ Gudur } & \multirow[t]{4}{*}{14.2} & \multirow[t]{4}{*}{8.8} & SFAP33 & 0.62 & & \\
\hline & & & & SFAP34 & 2.52 & & \\
\hline & & & & SFAP35 & 0.45 & & \\
\hline & & & & SFAP36 & 1.34 & & \\
\hline & \multirow[t]{4}{*}{ Kodimur } & \multirow[t]{4}{*}{10.3} & \multirow[t]{4}{*}{9.4} & SFAP37 & 2.01 & & \\
\hline & & & & SFAP38 & 0.11 & & \\
\hline & & & & SFAP39 & 1.87 & & \\
\hline & & & & SFAP40 & 2.10 & & \\
\hline
\end{tabular}


Table.3 Per cent disease incidence of Tobacco streak virus (TSV) in cucumber samples and absorbance values (A405 $\mathrm{nm}$ ) of samples collected from different mandals of Karnataka

\begin{tabular}{|c|c|c|c|c|c|c|c|}
\hline \multirow[t]{2}{*}{ District } & \multirow[t]{2}{*}{ Place (Mandal) } & \multicolumn{2}{|c|}{ Per cent disease incidence } & \multirow{2}{*}{$\begin{array}{l}\text { Sample } \\
\text { code }\end{array}$} & \multirow[t]{2}{*}{$\left(\mathbf{A}_{405} \mathbf{n m}\right)$} & \multirow{2}{*}{$\begin{array}{l}\text { Range of Absorbance } \\
\text { values }\left(\mathbf{A}_{405} \mathrm{~nm}\right)\end{array}$} & \multirow{2}{*}{$\begin{array}{l}\text { Range of disease } \\
\text { incidence }(\%)\end{array}$} \\
\hline & & Kharif2014 & Kharif 2015 & & & & \\
\hline \multirow[t]{17}{*}{ Tumkur (Karnataka) } & \multirow[t]{4}{*}{ Sira } & \multirow[t]{4}{*}{22.8} & \multirow[t]{4}{*}{14.2} & CUKA1 & 2.62 & \multirow[t]{16}{*}{$0.28-3.18$} & \multirow[t]{16}{*}{$5-22$} \\
\hline & & & & CUKA2 & 1.28 & & \\
\hline & & & & CUKA3 & 0.52 & & \\
\hline & & & & CUKA4 & 1.43 & & \\
\hline & \multirow[t]{4}{*}{ Madhugiri } & \multirow[t]{4}{*}{13.2} & \multirow[t]{4}{*}{13.2} & CUKA5 & 0.86 & & \\
\hline & & & & CUKA6 & 1.91 & & \\
\hline & & & & CUKA7 & 0.37 & & \\
\hline & & & & CUKA8 & 2.44 & & \\
\hline & \multirow[t]{4}{*}{ Tiptur } & \multirow[t]{4}{*}{14.8} & \multirow[t]{4}{*}{9.8} & CUKA9 & 0.28 & & \\
\hline & & & & CUKA10 & 1.82 & & \\
\hline & & & & CUKA11 & 2.10 & & \\
\hline & & & & CUKA12 & 0.67 & & \\
\hline & \multirow[t]{4}{*}{ Gubbi } & \multirow[t]{4}{*}{21.2} & \multirow[t]{4}{*}{11.2} & CUKA13 & 3.18 & & \\
\hline & & & & CUKA14 & 0.32 & & \\
\hline & & & & CUKA15 & 1.62 & & \\
\hline & & & & CUKA16 & 0.28 & & \\
\hline & & & & & & & Contd... \\
\hline \multirow[t]{2}{*}{ District } & \multirow[t]{2}{*}{ Place (Mandal) } & \multicolumn{2}{|c|}{ Per cent disease incidence } & Sample & $\left(\mathbf{A}_{405} \mathbf{n m}\right)$ & Range of Absorbance & Range of disease \\
\hline & & Kharif2014 & Kharif 2015 & code & & values $\left(\mathbf{A}_{405} \mathbf{n m}\right)$ & incidence (\%) \\
\hline \multirow[t]{14}{*}{ Tumkur (Karnataka) } & \multirow[t]{4}{*}{ Pavagada } & \multirow[t]{4}{*}{19.8} & 5.2 & CUKA17 & 1.55 & $0.28-3.18$ & $5-22$ \\
\hline & & & & CUKA18 & 1.37 & & \\
\hline & & & & CUKA19 & 1.42 & & \\
\hline & & & & CUKA20 & 2.44 & & \\
\hline & Davanagere & 14,2 & 7.8 & CUKA21 & 1.28 & & \\
\hline & & & & CUKA22 & 2.62 & & \\
\hline & & & & CUKA23 & 1.62 & & \\
\hline & & & & CUKA24 & 2.46 & & \\
\hline & Harapanahalli & 10.9 & 9.2 & CUKA25 & 1.42 & & \\
\hline & & & & CUKA26 & 2.29 & & \\
\hline & & & & CUKA27 & 1.32 & & \\
\hline & & & & CUKA28 & 1.92 & & \\
\hline & & & & CUKA29 & 0.42 & & \\
\hline & & & & CUKA30 & 2.42 & & \\
\hline
\end{tabular}


Table.4 Detection of Tobacco streak virus (TSV) in sap inoculated cowpea seedlings (Vigna unguiculata cv. 132) by DAC-ELISA

\begin{tabular}{|l|l|}
\hline Virus isolates & $\left(\mathbf{A}_{405} \mathrm{~nm}\right)$ \\
\hline GNAP7 & $2.45-3.12$ \\
\hline SFAP17 & $2.01-2.82$ \\
\hline CUKA13 & $2.58-2.96$ \\
\hline PHAP15 & $2.34-2.67$ \\
\hline Positive control & 3.02 \\
\hline Negative control & 0.12 \\
\hline
\end{tabular}

Fig.1 Symptoms exhibited by Tobacco streak virus infected groundnut plants

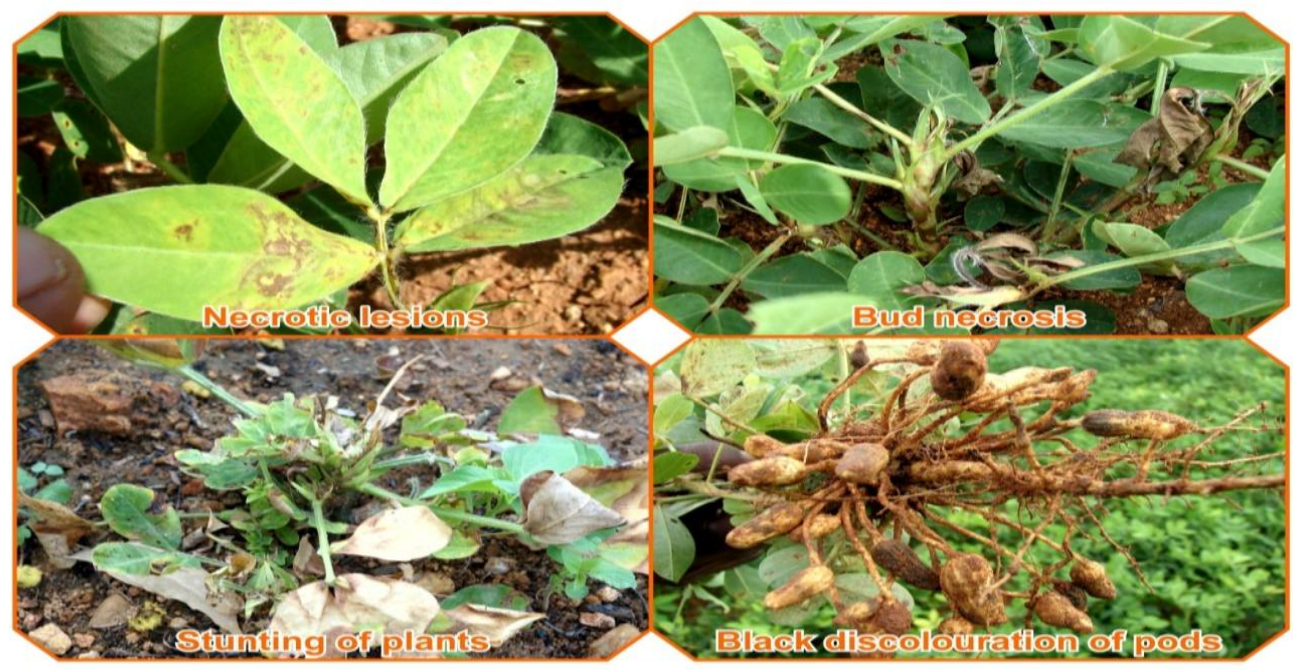

Fig.2 Symptoms exhibited by Tobacco streak virus infected sunflower plants

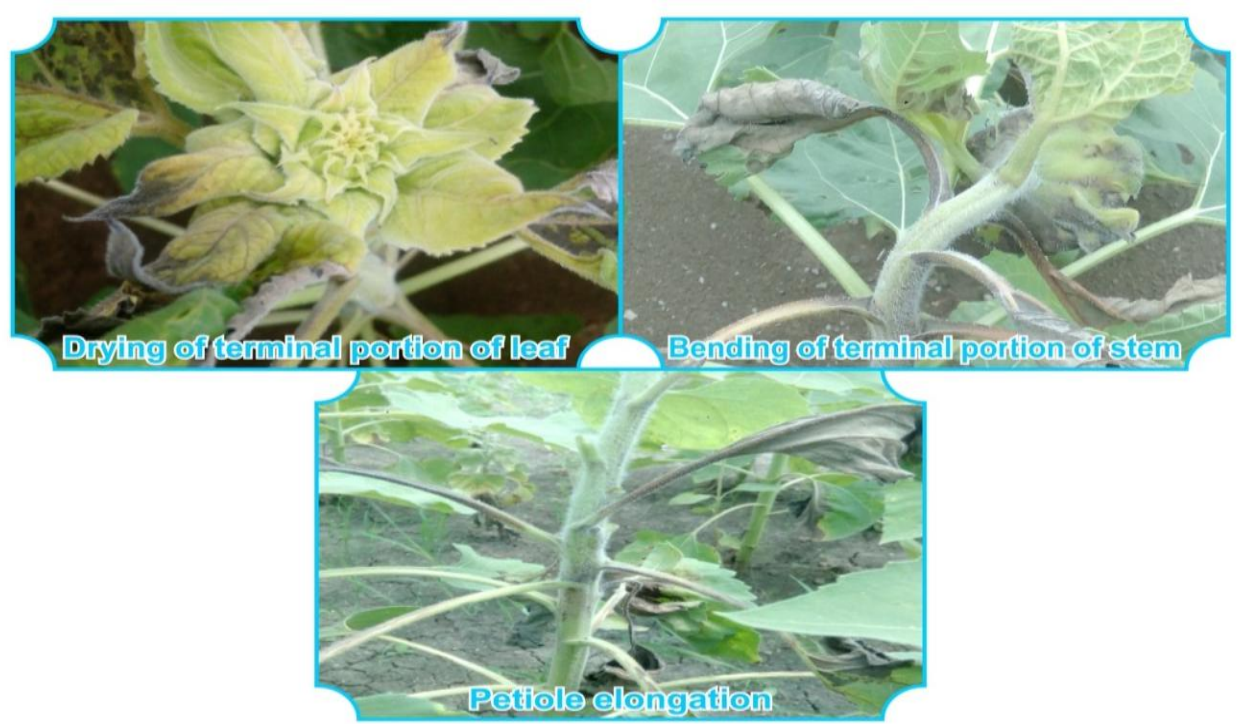


Fig.3 Symptoms exhibited by Tobacco streak virus infected cucumber plants
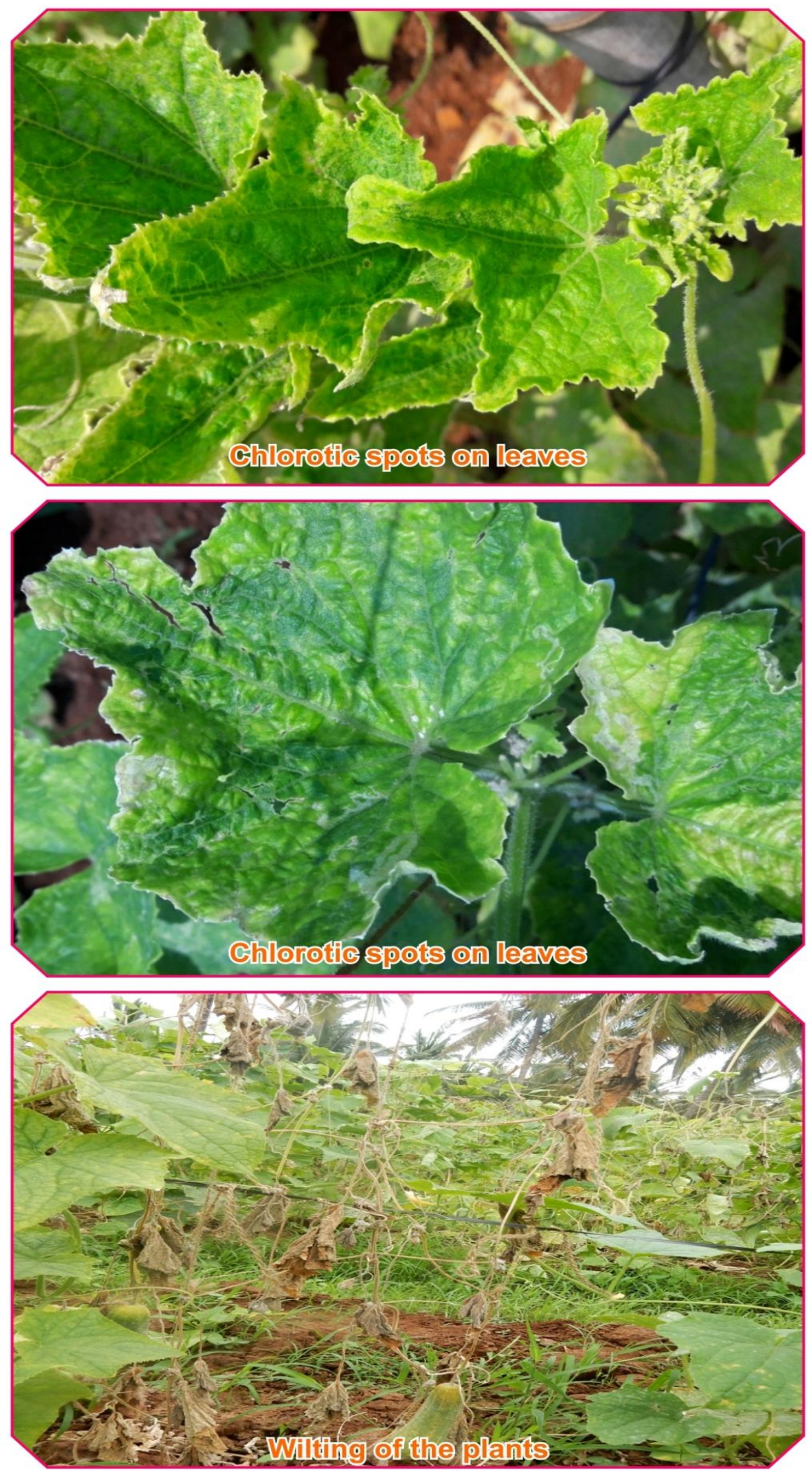
Fig.4 Maintenance of TSV isolates on test host, cowpea

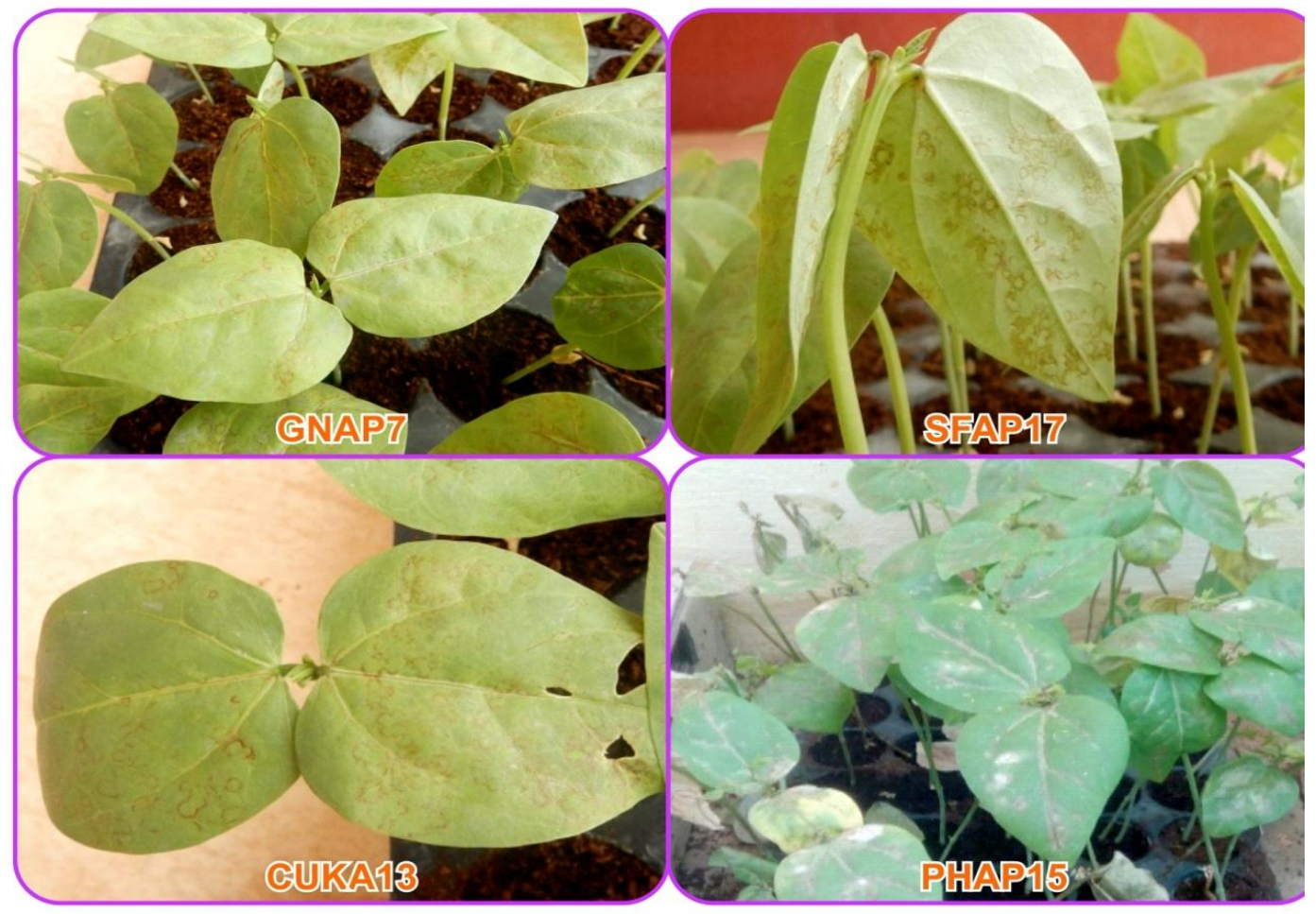

Fig.5 Manifestation of TSV symptoms on cowpea

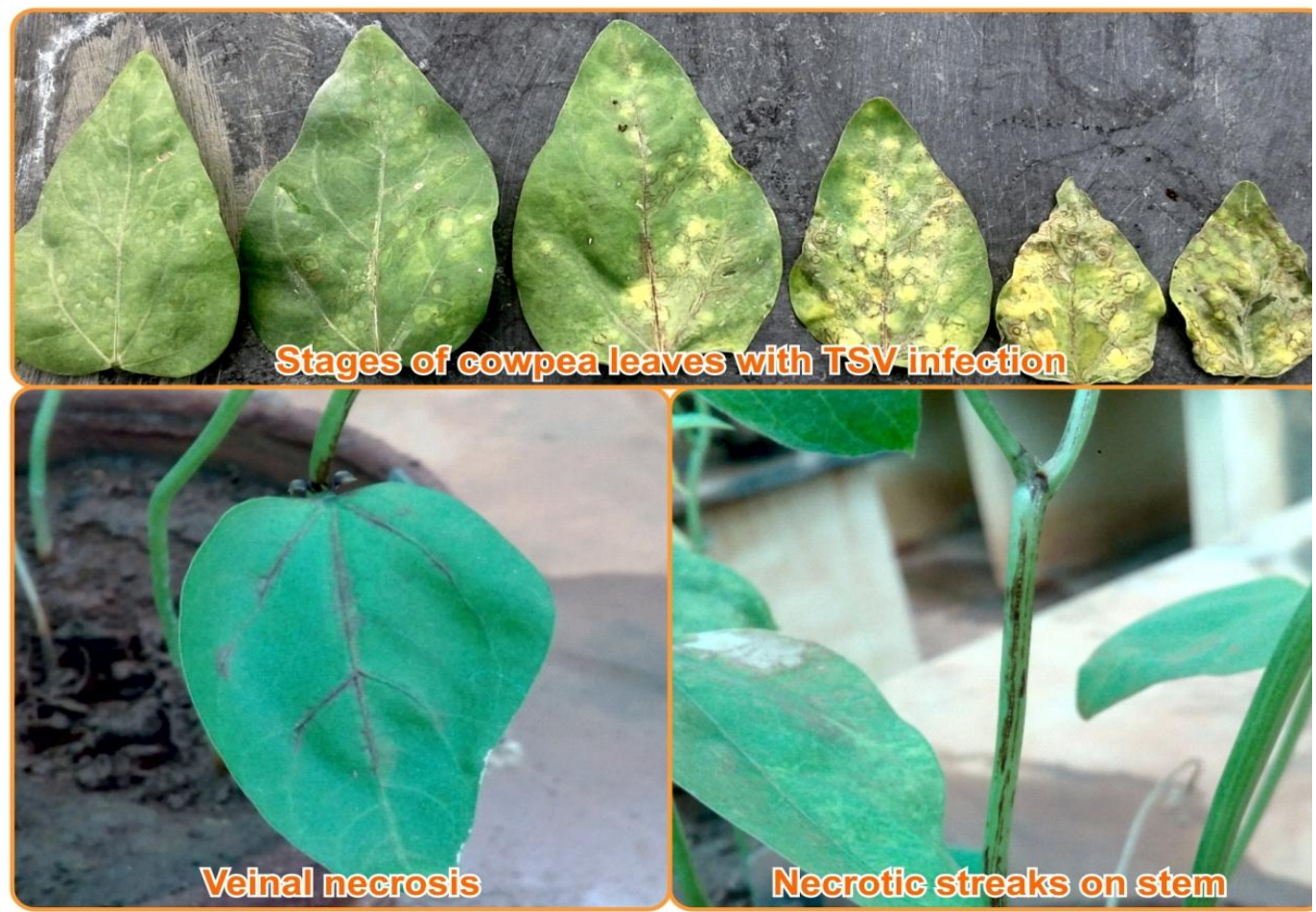


Localised symptoms were observed on cowpea after 1-2 Days Post Inoculation (DPI). Initial symptoms included pale necrotic spots followed by yellowing and wilting of inoculated leaves (Fig. 4 and 5). Similar symptoms were produced on healthy groundnut plants upon re inoculation using cowpea samples. Groundnut TSV isolate, GNAP7 maintained on cowpea was used for biological and molecular characterization.

Suspected TSV infected sunflower samples showing typical symptoms of necrotic streaks on stem and petioles, bending of terminal portion of stem, petiole elongation and drying of terminal portion of leaves were collected from farmer's fields of different mandals in Kurnool district. The collected samples were subjected to immuno-assay and bio-assay separately. Out of 40 samples collected, 25 samples reacted with polyclonal antiserum of TSV by DAC-ELISA. The absorbance values $\left(\mathrm{A}_{405} \mathrm{~nm}\right.$ ) ranged from 0.11-2.76 (Table 2). ELISA positive sunflower TSV samples were used for sap inoculation on cowpea seedlings (Vigna unguiculata cv. 132), a diagnostic host for TSV and inoculated on first true leaf stage seedlings (5 days old) and presence of virus was confirmed by DAC-ELISA (A $405 \mathrm{~nm}$ : 2.01-2.82) (Table 4). Pale necrotic symptoms were observed after 3 Days Post Inoculation (DPI). Later necrosis spread to petiole and lead to defoliation of cowpea leaves (Fig. 4 and 5). Similar symptoms were produced on healthy sunflower plants upon re inoculation using cowpea samples. Sunflower TSV isolate, SFAP17 maintained on cowpea was used further for biological and molecular characterization.

Cucumber plants showing symptoms of tip necrosis characterized by necrotic lesions on leaves and general leaf and stem necrosis extending to mid veins, petioles, flower buds and tip, were collected from farmer's fields of different mandals in Tumkur district of
Karnataka state. The samples were subjected to Immuno- and bio-assays separately. Out of 30 samples collected 24 samples reacted with polyclonal antiserum of TSV by DACELISA. The absorbance values $\left(\mathrm{A}_{405} \mathrm{~nm}\right)$ ranged from 0.28-3.18 (Table 3). TSV from symptomatic cucumber samples were sap transmitted to the cowpea seedlings (Vigna unguiculata cv.132) and presence of virus was confirmed by DAC-ELISA ( $\mathrm{A}_{405} \mathrm{~nm}$ : 2.58-2.96) (Table 4). Initially necrotic spots developed on leaves which later lead to drying and defoliation of leaves (Fig. 4 and 5). Similar symptoms were produced on healthy cucumber plants upon re inoculation using cowpea samples. Cucumber TSV isolate, CUKA13 maintained on cowpea was used for biological and molecular characterization.

In the present study TSV produced various symptoms on cowpea seedlings which included necrotic lesions, systemic veinal necrosis, necrotic streaks on stem, finally leading to complete drying of leaves. These results are supported by Ramaiah et al., (2001), Ladhalakshmi et al., (2006) and Arun Kumar et al., (2008) (circular necrotic lesions on cowpea are the characteristic symptoms of TSV by mechanical inoculation).

\section{References}

Anonymous, 2002. Annual Progress Report of AICRP on Oilseeds-Sunflower, 200203. Directorate of Oilseed Research, Hyderabad. 49

Arun Kumar, N., Lakshmi Narasu, M., Usha Zehr, B and Ravi, K.S. 2008. Molecular characterization of Tobacco streak virus causing soybean necrosis in India. Indian Journal of Biotechnology. 7(2): 14-217.

DOR Annual Report. 2001. Annual report (1999-2000). Directorate of Oilseed research, Hyderabad. 
Halakeri, A.V. 1999. Studies on sunflower necrosis disease. M.Sc. (Agri.) Thesis, University of Agricultural Sciences, Dharwad.

Krishna Reddy, M., Devaraj., Lakshmi, R., Salil, J and Samuel, D.K. 2003. Outbreak of Tobacco streak virus causing necrosis of cucumber (Cucumis sativus) and gherkin (Cucumis anguria) in India. Plant Dis. 87: 1264.

Ladhalakshmi, D., Ramiah, M., Ganapathy, T., Krishna Reddy, M., Khabbaz S.E., Merin Babu and Kamalakannan, A., 2006. First report of the natural occurrence of Tobacco streak virus on blackgram (Vigna mungo). Plant Pathology. 12: 55.

Prasada Rao, R.D.V.J., Reddy, D.V.R., Nigam, S.N., Reddy, A.S., Waliyar, F., Yellamanda Reddy, T., Subramanyam, K., Johnsudheer, M., Naik, K.S.S.,
Bandyopadhyay, A., Desai, S., Ghewande, M.P., Basu, M.S and Somasekhar. 2003. Peanut stem necrosis-A new disease of groundnut in India. Information Bulletin no. 67. ICRISAT, Patancheru, Andhra Pradesh, pp. 12.

Ramaiah, M., Bhat, A. I., Jain, R. K., Pant, R. P., Ahlawat, Y.S., Prabhakar, K and Varma, A. 2001. Isolation of an isometric virus causing sunflower necrosis disease in India. Plant Disease. 85: 443.

Reddy, A.S., Prasad Rao, R.D.V.J., Thirumala Devi, K., Reddy, S.V., Maya, M.A., Roberts, I., Satyanarayana, T., Subramaniam, K. and Reddy, D.V.R. 2002. Occurrence of Tobacco streak virus on peanut (Arachis hypogaea L.) in India. Plant Dis., 86:173-178.

\section{How to cite this article:}

Sunil Kumar M., R. Sarada Jayalakshmi Devi, M. Krishna Reddy, K. Vemana, T. Murali Krishna and Prasanthi, L. 2018. Survey for the Incidence of Tobacco streak virus (TSV) in Field and Horticultural Crops. Int.J.Curr.Microbiol.App.Sci. 7(10): 3657-3669. doi: https://doi.org/10.20546/ijcmas.2018.710.423 